\title{
Estimating Receiver Operating Characteristic Curves with Covariates When There Is No Perfect Reference Test for Diagnosis of Johne's Disease
}

\author{
C. Wang, ${ }^{*}$ B. W. Turnbull, ${ }^{*}$ Y. T. Gröhn, $\dagger^{1}$ and S. S. Nielsen‡ \\ *Department of Statistical Science, Cornell University, Ithaca, NY 14853 \\ †Department of Population Medicine and Diagnostic Sciences, College of Veterinary Medicine, Cornell University, Ithaca, NY 14853 \\ $\ddagger$ Department of Large Animal Sciences, The Royal Veterinary and Agricultural University, Frederiksberg, Denmark
}

\begin{abstract}
Paratuberculosis (Johne's disease) is a significant animal health problem. Evaluation of diagnostic tests for Johne's disease has been difficult due to lack of a gold standard test. In recent years, there has been interest in receiver operating characteristic (ROC) curve estimation without any gold standard test. Typically, either Bayesian or maximum likelihood methods are proposed. Although these methods overcome the lack of a gold standard test in ROC curve estimation, little work has been done to incorporate covariates in the analysis. In this paper, we propose a method for estimation of ROC curves based on statistical models to adjust for covariate effects when the true disease states of test animals are unknown. The covariates may be correlated with the disease process or with the diagnostic testing procedure, or both. We propose a 2-part Bayesian model: first, a logistic regression model for disease prevalence is used to fit the covariates; second, a linear model is used to fit the covariates to the distribution of test scores. We used Markov chain Monte Carlo methods to compute the posterior estimates of the sensitivities and specificities that provide the groundwork for inference concerning the diagnostic procedure's accuracy. We applied the methodology to milk ELISA scores from several dairy-cow herds for the diagnostic testing of paratuberculosis. We found that both milk yield and its interaction with age had significant effects on the disease process whereas only milk yield was significant on the testing procedure.
\end{abstract}

Key words: Bayesian method, Johne's disease, milk yield, receiver operating characteristic curve

\section{INTRODUCTION}

Paratuberculosis (Johne's disease) is a severe chronic progressive gastroenteritis in ruminants caused by $M y$ -

Received December 20, 2005.

Accepted February 28, 2006.

${ }^{1}$ Corresponding author: ytg1@cornell.edu cobacterium avium ssp. paratuberculosis (basonym Map). Johne's disease is a major animal health problem (Rideout et al., 2003), which needs to be studied further, so as to control it more effectively. Control of Johne's disease has been difficult because of the lack of accurate diagnostic tests. Tests such as milk ELISA and fecal culture are known to be somewhat inaccurate. There is a great need for specific and sensitive tests for detecting early infections of Johne's disease. However, when no gold standard (GS) tests are available for comparison, the performances of both existing imperfect tests and those yet to be proposed are difficult to evaluate.

For diagnostic tests with binary outcomes, sensitivity (Se) and specificity (Sp) are used to characterize the accuracy of a diagnostic test. Receiver operating characteristic (ROC) curves can be used to assess the accuracy of ordinal or continuous-scaled diagnostic tests by plotting all possible pairs of sensitivity and 1 - specificity values. Receiver operating characteristic curves enable the assessment of tests without specifying a particular threshold value. In particular, area under the curve (AUC) is an important feature because it provides an overall measure of accuracy for the test. Principles of ROC curve estimation using parametric and nonparametric methods were reviewed previously by Zweig and Campbell (1993) and Greiner et al. (2000).

An important assumption of traditional ROC analysis is that a GS reference test with perfect Se and Sp is available for a test data set. However, a GS test may be expensive, or may not exist. Therefore, imperfect diagnostic tests are frequently used in veterinary, medical, and epidemiological research. Less work has been done on estimating ROC without GS tests (reviewed by Pepe, 2003).

For binary outcome test data, Hui and Walter (1980) used maximum likelihood methods to estimate sensitivity and specificity when a GS test was not available. Garrett et al. (2002) proposed a latent class approach for evaluating the performance of independent tests in the absence of GS data with Markov chain Monte Carlo (MCMC) method. Joseph et al. (1995) developed Bayesian methods for evaluating conditionally independent 
diagnostic tests without a GS test. Dendukuri and Joseph (2001) and Georgiadis et al. (2003) generalized Joseph et al.'s (1995) work to evaluate dependent tests, also without a GS test.

For ordinal or continuous outcome test data, construction of a ROC curve requires all possible pairs of sensitivity and 1 - specificity values to be estimated. When no GS test is available, these pairs can be estimated separately using the method of Hui and Walter (1980); however, as shown by Nielsen et al. (2002a), the resulting ROC curves may be nonmonotone. Wang et al. (2006) developed a Bayesian methodology for nonparametric estimation of ROC curves without a GS test, which does assure the natural monotonicity property of the ROC curve estimate. For the situation in which the test values (or transformed test values) of both diseased and nondiseased individuals have continuous normal distributions, Choi et al. (2006) proposed a Bayesian method for estimating an ROC curve when there is no GS test.

When covariate information is available, it can be used to increase the effectiveness of continuous markers in distinguishing between healthy and diseased subjects; Pepe (2003) discussed covariate effects on tests and the need to identify them. Faraggi (2003) and Schisterman et al. (2004) developed parametric methods to estimate ROC curves and derive approximate confidence intervals for the corresponding AUC when markers were adjusted for covariates. However, when there is no GS test, there is no statistical methodology currently available to adjust the ROC curve for covariates.

As discussed in Nielsen et al. (2002b), van Schaik et al. (2003), and Nielsen and Toft (2006) among others, prevalence of Johne's disease is usually observed to depend on covariates, such as age and milk yield. Kudahl et al. (2004) have studied the relationship between paratuberculosis and these variables and also between ELISA test scores and these variables. In this study, our objectives were 2-fold: The first objective was to develop statistical methodology to estimate the effects of the covariates on the ROC curve and correct for them when appropriate; the second objective was to apply this statistical methodology to Johne's disease data in cows.

\section{MATERIALS AND METHODS}

\section{Two-Part Statistical Model}

Herein we propose statistical methodology for assessing the accuracy of the ELISA test (Test 1) by comparing it to the imperfect fecal culture reference test (Test 2), and correcting for covariate effects. Failing to adjust for covariate effects could cause a biased estimate of the ROC curve for the ELISA test.
Suppose we have a sample of $n$ animals. For the $i$ th animal $(i=1, \ldots n)$, let $D_{i}(=0$ or 1$)$ denote the true unknown binary disease status, $T_{i}$ denote the value of Test 1, measured on a continuous scale, possibly transformed, and let $R_{i}=1$ (positive) or 0 (negative) denote the diagnostic value of Reference Test 2 , which is assumed measured as a binary indicator. If the scale of this reference test is ordinal or continuous, it can be dichotomized using a cutoff value for classification. The focus here is to estimate the distribution of Test 1 scores conditional on the true disease status; i.e., $T_{i} \mid D_{i}$. We assume the 2 tests are conditionally independent; i.e., $T_{i}$ and $R_{i}$ are independent given disease status $D_{i}$.

For the $i$ th individual, we also have $K$ measured covariates $\mathrm{Z}_{i, 1}, \ldots, Z_{i, K}$, which could affect either the disease status $D_{i}$ or the Test 1 scores given the disease status, or both. Denote $\mathbf{Z}_{i}=\left(1, Z_{i, 1}, \ldots, Z_{i, K}\right)^{\prime}$, which is a $K+1$ dimensional vector. To model the covariate effects on the disease process, we used a logistic model for the odds ratio:

$$
\log \frac{\operatorname{Pr}\left(D_{i}=1\right)}{1-\operatorname{Pr}\left(D_{i}=1\right)}=\delta_{0}+\delta_{1} Z_{i, 1}+\ldots+\delta_{K} Z_{i, K} .
$$

The model assumes that this log-odds (or logit) is a linear function of the covariates $\mathbf{Z}_{i}$. Here, $\delta_{1}, \ldots, \delta_{K}$ are unknown regression coefficients, and $\delta_{0}$ is the baseline intercept. Denote $\delta=\left(\delta_{0}, \delta_{1} \ldots, \delta_{K}\right)^{\prime}$.

To model the effects of the covariates on the Test 1 scores given disease status, we used a linear model for the conditional mean of the Test 1 scores.

$$
\begin{aligned}
& E\left(T_{i} \mid D_{i}=1\right)=\beta_{D, 0}+\beta_{D, 1} Z_{i, 1}+\ldots+\beta_{D, K} Z_{i, K} \\
& E\left(T_{i} \mid D_{i}=0\right)=\beta_{H, 0}+\beta_{H, 1} Z_{i, 1}+\ldots+\beta_{H, K} Z_{i, K} .
\end{aligned}
$$

Here, $\boldsymbol{\beta}_{D}=\left(\beta_{D, 0}, \beta_{D, 1}, \ldots, \beta_{D, K}\right)^{\prime}$ and $\boldsymbol{\beta}_{H}=\left(\beta_{H, 0}, \beta_{H, 1}, \ldots\right.$, $\left.\beta_{H, K}\right)^{\prime}$ are the unknown regression coefficients for diseased and healthy individuals, respectively. By proposing 2 sets of coefficients of covariates for diseased and healthy groups separately, we were able to capture nonuniform covariate effects, a more general assumption (see Schisterman et al., 2004). In our analysis, we assumed the conditional Test 1 values to be normally distributed with variances $\operatorname{Var}\left(T_{i} \mid D_{i}=1\right)=\sigma_{D}^{2}$ and $\operatorname{Var}\left(T_{i} \mid D_{i}=0\right)=\sigma_{H}^{2}$. However, this is not essential to our method; other distributional assumptions can be made.

For the imperfect binary Reference Test 2, we have the natural misclassification model for the diagnostic values $\left\{R_{i} ; i=1, \ldots, n\right\}$ :

$$
\begin{gathered}
\left(R_{i} \mid D_{i}=1\right) \sim \operatorname{Bin}\left(1,1-\alpha_{D}\right) \\
\left(R_{i} \mid D_{i}=0\right) \sim \operatorname{Bin}\left(1, \alpha_{H}\right),
\end{gathered}
$$


where $\alpha_{D}$ and $\alpha_{H}$ are, respectively, the false negative probability and the false positive probability for Test 2 . These parameters are also unknown and will be estimated along with the parameters of the Test 1 model. The joint likelihood of the disease status and the Test 1 and Test 2 outcomes for the $i$ th individual is:

$$
\begin{aligned}
& L\left(T_{i}, R_{i}, D_{i} \mid \boldsymbol{Z}_{i}, \boldsymbol{\beta}_{D}, \boldsymbol{\beta}_{H}, \sigma_{D}^{2}, \sigma_{H}^{2}, \alpha_{D}, \alpha_{H}, \boldsymbol{\delta}\right)= \\
& \left\{\frac{1}{\sqrt{2 \pi} \sigma_{D}} \exp \left[\frac{-\left(T_{i}-\boldsymbol{\beta}_{D}^{\prime} \cdot \mathbf{Z}_{i}\right)^{2}}{2 \sigma_{D}^{2}}\right]\right\}^{D_{i}} \\
& \left\{\frac{1}{\sqrt{2 \pi} \sigma_{H}} \exp \left[\frac{-\left(T_{i}-\boldsymbol{\beta}_{H}^{\prime} \cdot \mathbf{Z}_{i}\right)^{2}}{2 \sigma_{H}^{2}}\right]\right\}^{1-D_{i}} \\
& \times\left[\alpha_{D}^{1-R_{i}}\left(1-\alpha_{D}\right)^{R_{i}}\right]^{D_{i}} \times\left[\alpha_{H}^{R_{i}}\left(1-\alpha_{H}\right)^{1-R_{i}}\right]^{1-D_{i}} \\
& \times\left[\frac{\exp \left(\delta^{\prime} \cdot \mathbf{Z}_{i}\right)}{1+\exp \left(\delta^{\prime} \cdot \mathbf{Z}_{i}\right)}\right]^{D_{i}}\left[\frac{1}{1+\exp \left(\delta^{\prime} \cdot \mathbf{Z}_{i}\right)}\right]^{1-D_{i}}
\end{aligned}
$$

The overall joint likelihood for the sample is a product of the term in equation [1] over $i=1, \ldots, n$. A detailed examination of the joint likelihood shows that the model parameters are identifiable.

To estimate the parameters in the model, one approach would be to use maximum likelihood. Because the $\left\{D_{i} ; i=1, \ldots, n\right\}$ are unobserved, one might consider the use of an expectation maximization (EM) algorithm to maximize the likelihood with respect to all parameters plus the $\left\{D_{i} ; i=1, \ldots, n\right\}$. This approach was proposed by Zhou et al. (2005) in the case of ordinal outcomes and no covariates. However, in our situation with moderate and large size data sets with covariates, the likelihood maximization becomes intractable. Instead, we took a Bayesian approach to estimation, which can be implemented in practice.

\section{The Bayesian Approach}

We estimated the parameters in the model by using Bayesian methods. Independent prior distributions are used for $\boldsymbol{\beta}_{D}, \boldsymbol{\beta}_{H}, 1 / \sigma_{D}^{2}, 1 / \sigma_{H}^{2}, \alpha_{D}, \alpha_{H}$, and $\delta$. Specifically, we assumed normal $\mathrm{N}(0,1000)$ for each of $\beta_{D, 0}, \beta_{D, 1}, \ldots$, $\beta_{D, K}, \quad \beta_{H, 0}, \quad \beta_{H, 1}, \quad \ldots, \quad \beta_{H, K}, \quad$ and $\delta_{0}, \delta_{1}, \ldots, \quad \delta_{K}$; gamma $(0.001,0.001)$ for $1 / \sigma_{D}^{2}$ and $1 / \sigma_{H}^{2}$; and beta $(0.5,0.5)$ for $\alpha_{D}$ and $\alpha_{H}$. These priors approximate the Jeffreys priors (Jeffreys, 1961) and were used as noninformative priors. Instead, informative priors could be used if previous knowledge is available for any of the parameters.

Based on the prior distributions and the likelihood of the observed data $\left\{T_{i} ; i=1, \ldots, n\right\},\left\{R_{i} ; i=1, \ldots, n\right\}$, the posterior distribution of the parameters together with disease status $\left\{D_{i} ; i=1, \ldots, n\right\}$ can be simulated by using
MCMC methods (see Robert and Casella, 2004). In particular, we used the Gibbs sampler and MetropolisHasting algorithm. The values in the chain are updated by Gibbs sampling from the full conditional distributions. Details are given in the Appendix. The model was programmed in MATLAB version 7.0 (The MathWorks, Inc., Natick, MA). The source code is available upon request from the authors. Alternatively, WinBUGS (Spiegelhalter et al., 1995) software may also be used.

\section{Application}

The cross-sectional data set used here came from part of a multipurpose study on infectious disease conducted in 1998 in Southern Jutland, Denmark, by the Danish Dairy Board (Andersen et al., 2000). These are the same data as used by Nielsen et al. (2002a). From 55 herds in the data set, 2,481 cows with completed information, including milk ELISA and fecal culture scores were used in the analysis. The milk ELISA test readings were analyzed based on corrected optical density measurements (Nielsen, 2002). A log-transformation was applied to account for the skewness of the readings and for the fact that the variance of the corrected optical density values increases with increasing mean values. The effects of covariates milk yield (kg), age (yr), and their interaction (milk $\times$ age) were estimated, and tested for significance.

\section{RESULTS AND DISCUSSION}

\section{Descriptive Findings}

We considered 4 models: 1) ignoring the covariate effects; 2) with covariate effects only affecting disease prevalence; 3 ) with covariate effects only affecting test outcome values; and 4) with covariate effects affecting both parts of the model specification. The fourth model is the most general and it includes the first 3 by restricting the corresponding covariate coefficients to be zero.

The MCMC method produces the posterior density of each parameter of interest (e.g., the regression coefficient). Successive values of each of the parameters are generated by simulation and the steady-state distribution is the posterior for that parameter (e.g., Robert and Casella, 2004). Figures 1 and 2 show the MCMC chain sample values for the regression coefficients of the fourth model; for example, assuming that the covariates affect both parts. The figures show that steady state has been reached after the burn-in period of 2,000 iterations; the further 3,000 iterations are typically considered enough to give an accurate estimate of the posterior distribution. More quantitative assessment of the convergence can also be used to justify this choice; for 


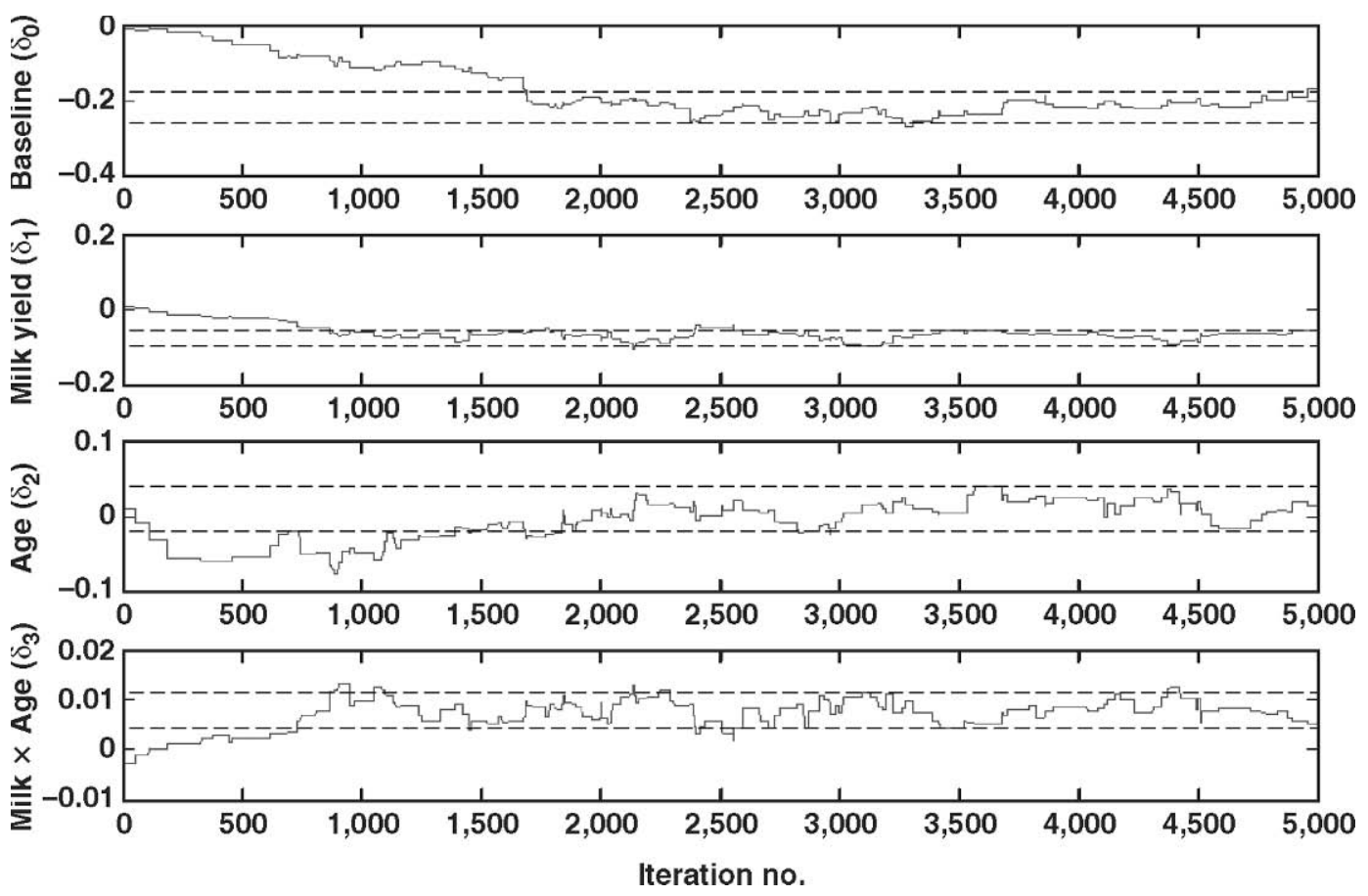

Figure 1. Markov chain Monte Carlo iterates as estimates for the coefficients of covariates relating to disease prevalence for Model 4 using the Danish Dairy Board data set. The horizontal dashed lines indicate the $2.5 \%$ and $97.5 \%$ quantiles of the posterior samples, which give the estimated $95 \%$ credible intervals.

example, CODA software (Best et al., 1995). Figures 1 and 2 are for model 4 ; the analogous plots for the other models show similar features. The bands in the figures are the estimated $95 \%$ credible intervals based on the posterior samples. The parameter estimates and corresponding credible intervals for all 4 models are shown in Tables 1 and 2 . The effects of covariates are statistically significant if the corresponding credible intervals do not include zero. In particular, for the fourth model, the effects of milk yield and the interaction milk $\times$ age were found to be significant for the disease prevalence specification, whereas only milk yield was found to have significant effects on the test outcome (ROC) part. To obtain final estimates of these significant effects, the fourth model was run again, this time restricting the nonsignificant regression coefficients in the ROC part to be zero. We label this fifth model as the "adjusted" model, and we term the first model (1) as the "unadjusted" model. We now compare these 2 models in more detail. This shows the difference that inclusion of covariates can bring, and illustrates the need to adjust for covariates.

\section{Covariate Effects on ROC Curves and Related Indices}

We investigated the covariate effects on the test performance in terms of ROC curves and related indices.
A commonly used measure of marker accuracy is the AUC of the ROC curve, which indicates an effective marker if it has values close to 1 . Another frequently used summary index is the Youden index (Youden, 1950), which is defined as $\max (\mathrm{Se}+\mathrm{Sp}-1)$ over all pairs of Se and Sp on the ROC curve. The critical threshold, $\mathrm{C}^{*}$, which corresponds to the maximum of $(\mathrm{Se}+\mathrm{Sp}$ -1) in the Youden index, may be used as a criterion to determine the cutoff value of the marker.

For the unadjusted model, in which normality is assumed and covariate effects are ignored, the estimated ROC curve is shown in the top panel of Figure 3. The estimated area under the ROC curve was 0.7279 , Youden index was 0.4287 , and the critical threshold $\mathrm{C}^{*}$ was -0.8303 .

For the adjusted model, the ROC curve was related to the covariate milk yield. The level of milk yield is capable of affecting the optical density in terms of dilution, which explains the negative signs of the estimated coefficients of this effect. It can be speculated that the dilution effects of milk yield were stronger on diseased cows, which have greater optical density than healthy cows. Another possible explanation is that cows with high levels of production of antibodies often have reduced milk yield, as demonstrated in Kudahl et al. (2004). The interaction between the effects and disease status implies that the test performance is different with different levels of milk yield. The estimated ROC 

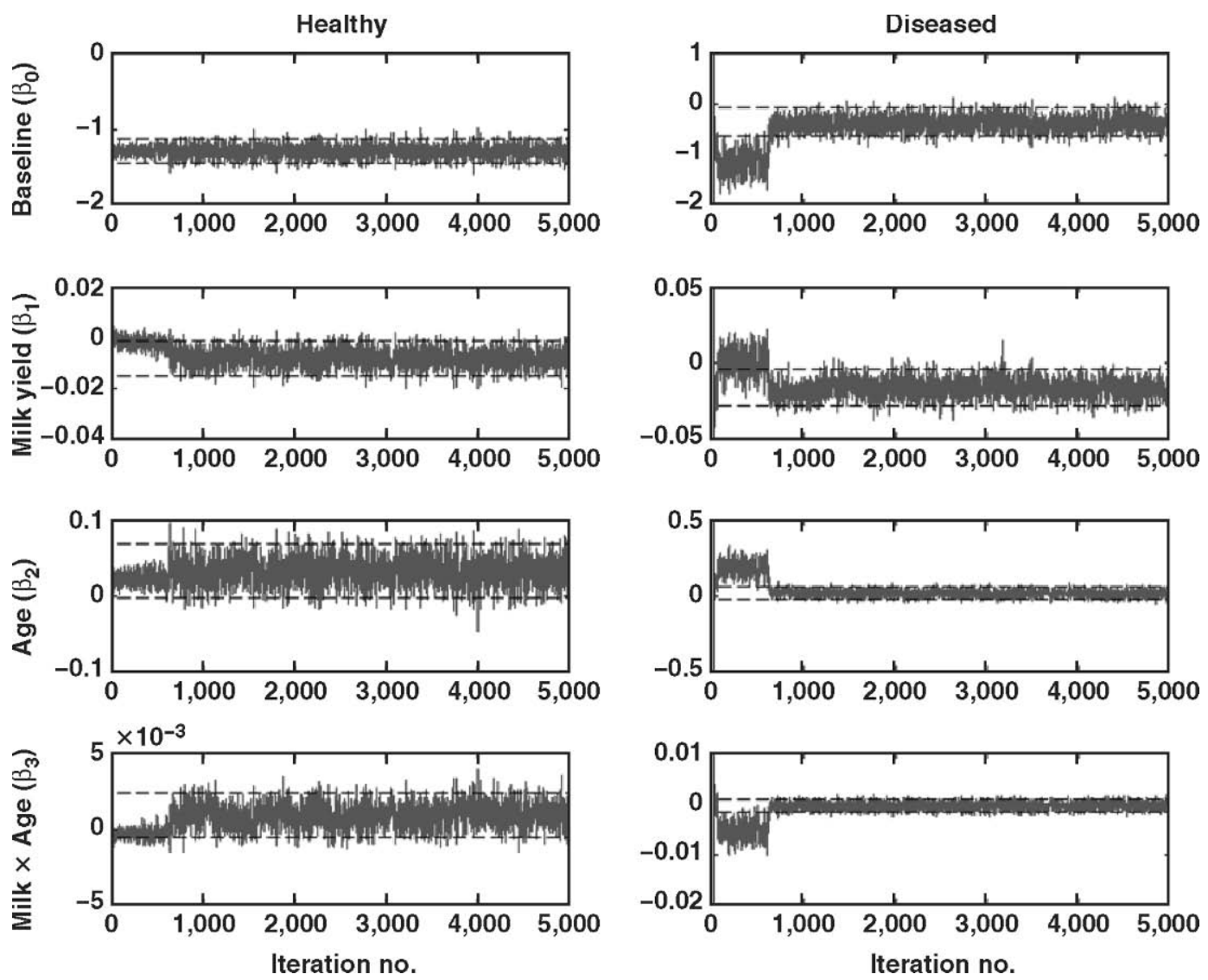

Figure 2. Markov chain Monte Carlo iterates as estimates for the coefficients of covariates on ELISA test scores for Model 4 using the Danish Dairy Board data set. Left panel: coefficients $\left\{\boldsymbol{\beta}_{H}\right\}$; right panel $\left\{\boldsymbol{\beta}_{D}\right\}$. The horizontal dashed lines indicate the $2.5 \%$ and $97.5 \%$ quantiles of the posterior samples, which give the estimated $95 \%$ credible intervals.

curves are presented in the bottom panel of Figure 3 for milk yields of 0,20 , and $40 \mathrm{~kg}$ (with AUC 0.8585 , 0.8095 , and 0.7513 , respectively). It can be seen that the test performs more poorly as the level of milk yield increases, which can be explained by the interaction between milk yield and disease status. However, comparing the bottom panel with the top panel of Figure 3 , it can be seen that the adjusted ROC curves with milk yield 0,20 , and $40 \mathrm{~kg}$ all perform better than the unadjusted ROC. This gain of test performance can be further confirmed by comparing the related indices. The adjusted estimates of the AUC, Youden index, and critical threshold value $\mathrm{C}^{*}$ as functions of milk yield are plotted in the top, middle, and bottom panels of Figure 4, respectively. Also plotted in these panels are the values of the corresponding estimated indices, which are constant when the covariate effects are ignored (Model 1). The adjusted estimates of AUC and Youden index dominate the corresponding unadjusted ones, except when the level of milk yield is extremely high $(>47)$.

\section{Covariate Effects on Disease Prevalence}

We then investigated the covariate effects on the disease prevalence. In the model (4) where the covariates could affect both parts, the estimated regression coefficients for milk yield, age, and milk $\times$ age were negative, positive, and positive, respectively. The effect of the interaction milk $\times$ age was found to be statistically significant, which suggests that all 3 effects should be included in the analysis, following common statistical practice. The positive effect of age is natural because older cows are more likely to become diseased. It is important to note that only lactating cows are included in the data set (no calves). Also, diseased cows generally produce less milk, which explains the negative effect of milk yield on disease prevalence. The interaction milk $\times$ age is intuitive because it is known that the milk yields of cows vary according to their ages.

\section{CONCLUSIONS}

Individual cows in the database were heterogeneous and it was recognized that information on covariates 
Table 1. Parameter estimates and corresponding 95\% credible intervals for ELISA test score for Models 1 to 4 using the Danish Dairy Board data set

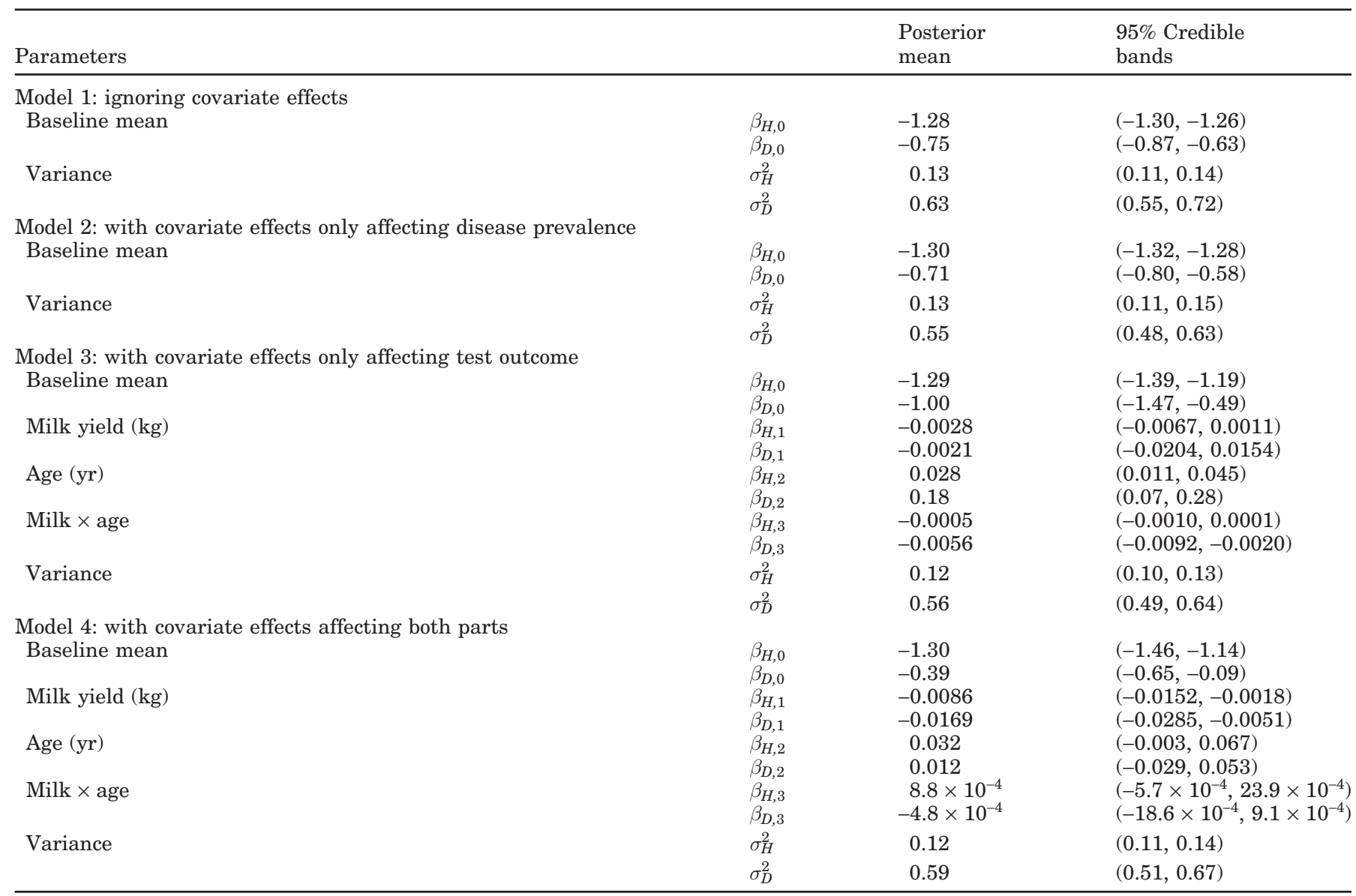

(age, milk yield, etc.) could help in making a diagnosis and hence, affect the evaluation of diagnostic tests. The previous literature on estimating ROC curves in the presence of covariates has relied on the existence of a GS test; therefore, in that situation, the only issue was the effect of covariates on the ROC curve. When the disease status of each subject cannot be ascertained perfectly, the covariate values must also be used to glean information about disease status of the individual cow. Both aspects of the effect of covariates need to be

Table 2. Parameter estimates and corresponding 95\% credible intervals for log odds ratio of disease prevalence for Models 1 to 4 using the Danish Dairy Board data set

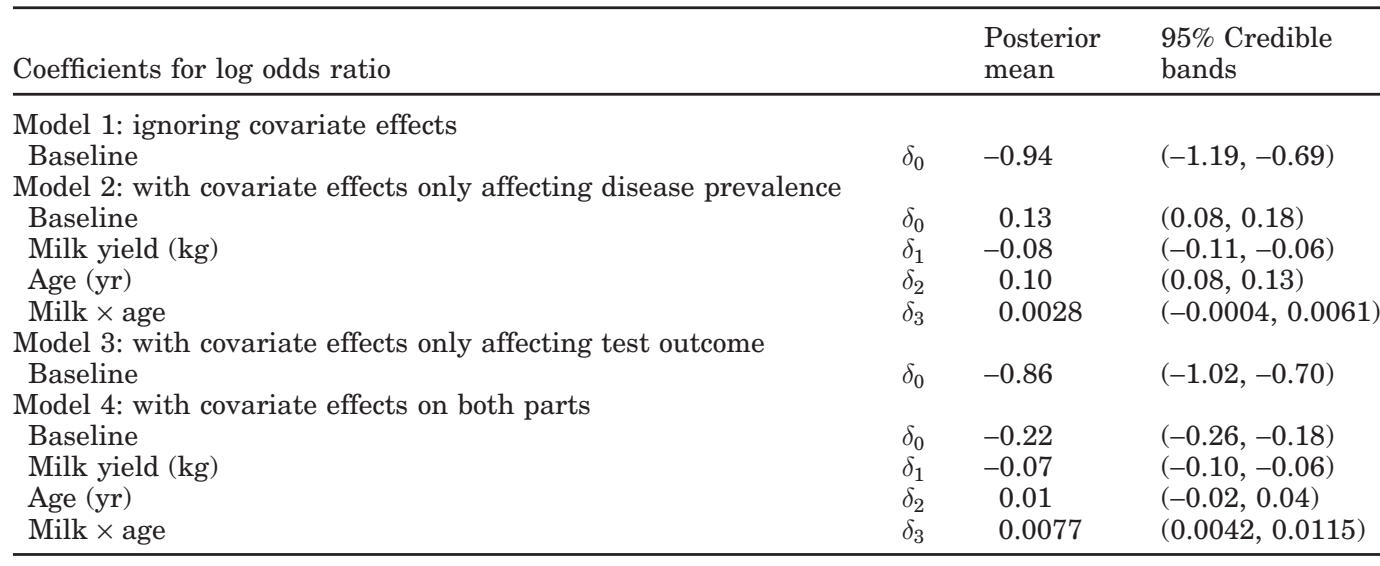



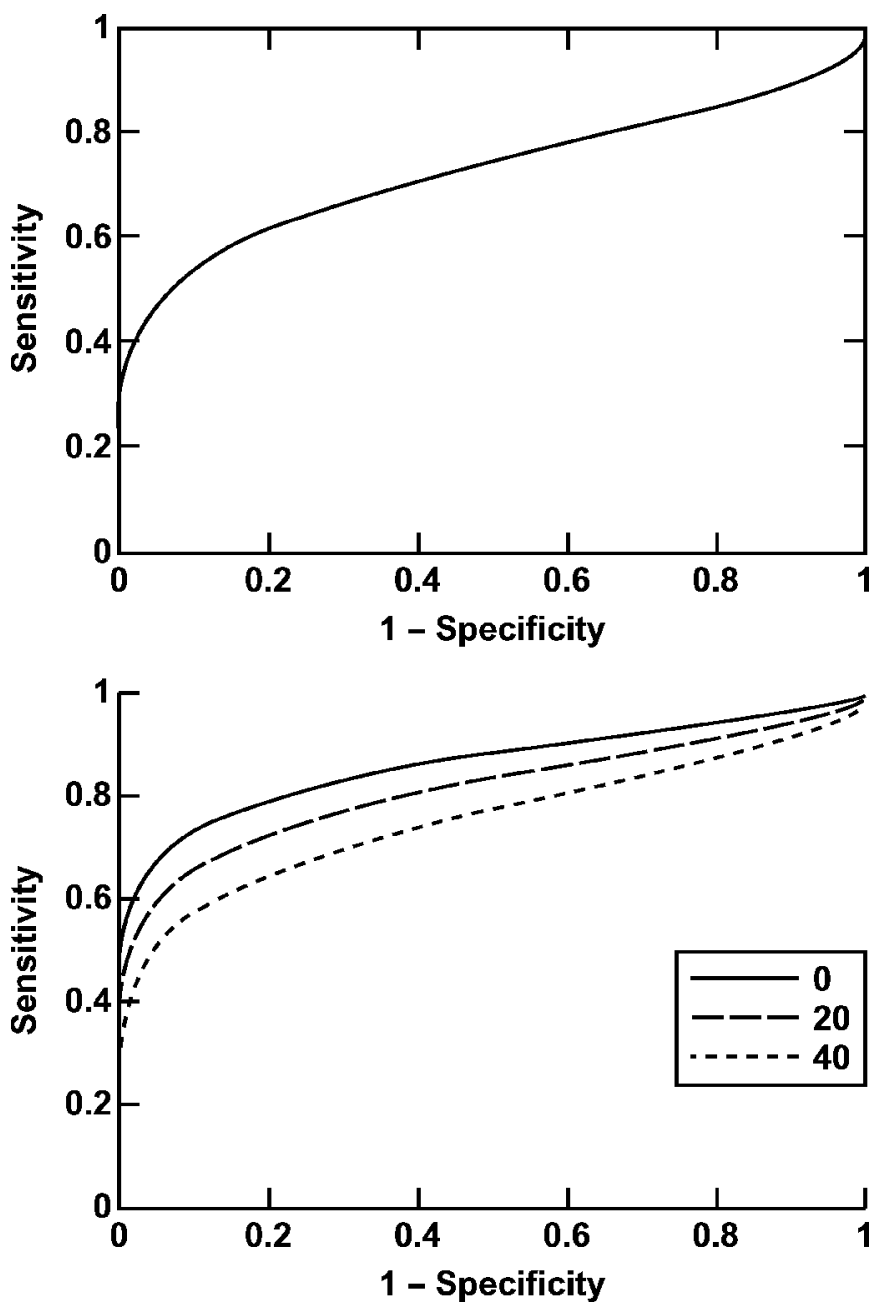

Figure 3. Estimated receiver operating characteristic curves of ELISA measurements using the Danish Dairy Board data set. Curve in top panel is for Model 1, without adjusting for covariates; curves in bottom panel are for Model 4, with hypothetical milk yield values of $0(-), 20(--)$ and $40 \mathrm{~kg}(\cdots)$

considered to provide a valid evaluation of a proposed new diagnostic test procedure. Our proposed 2-part statistical model and methods have provided a means to do this, as demonstrated by our application.

In our data set, we found that both milk yield and its interaction with age have significant effects on the disease status aspect, whereas only milk yield was significant for test response. These findings can be naturally explained by knowledge of the disease and the testing procedure. Adjusting for covariate effects was advantageous and necessary for estimating ROC curves without a GS test. It was shown that the estimated ROC curves and related indices were much better than the unadjusted one. These covariate-adjusted ROC curves could be used in conjunction with economic con-
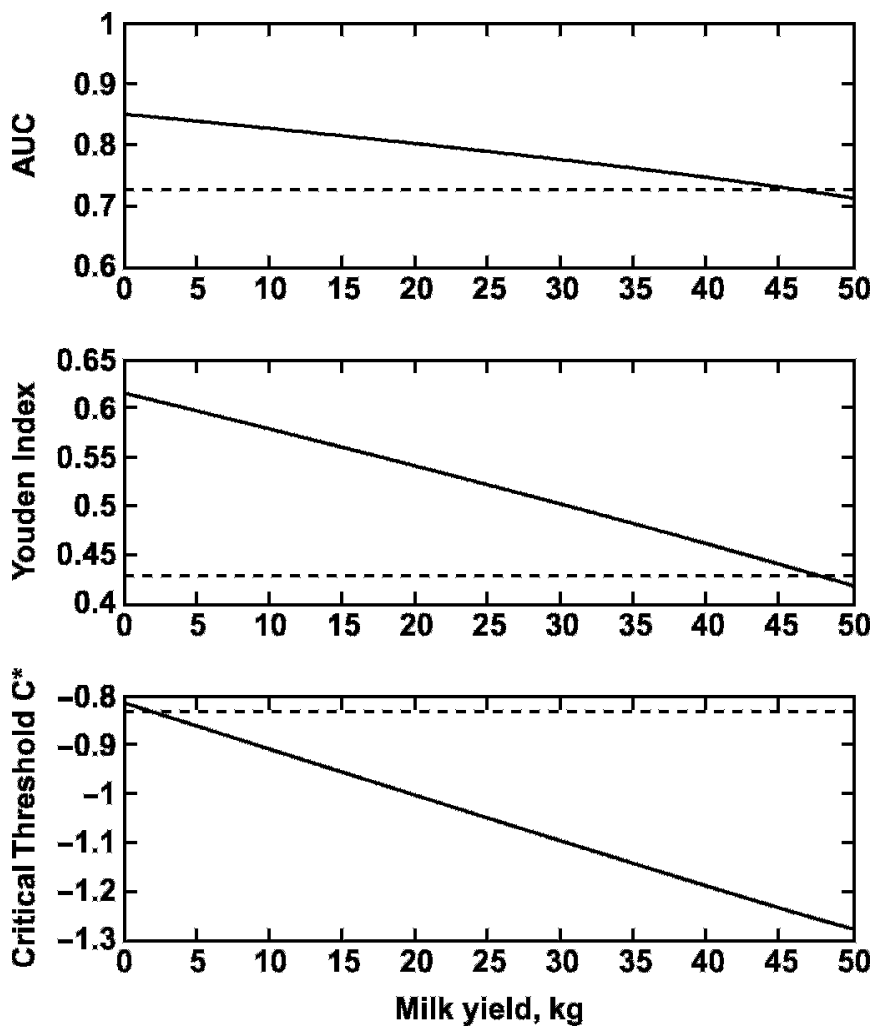

Figure 4. Estimated indices for receiver operating characteristic curve as functions of milk yield ( $\mathrm{kg}$ ), for Model $1(\cdots)$ ) and Model 4 (-). Top panel = estimated area under the curve (AUC); middle panel $=$ estimated Youden Index; bottom panel $=$ estimated critical threshold value, $\mathrm{C}^{*}$, for Youden Index.

siderations of error rates to set differing thresholds for diagnostic decisions for individual cows.

\section{ACKNOWLEDGMENTS}

This study was funded in part by grant USDACSREES/2004-35605-14243, JDIP: Johne's Disease Integrated Program in Research, Education, and Extension, and in part by grant R01 CA66218 from the National Institutes of Health.

\section{REFERENCES}

Andersen, H. J., K. Aagaard, F. Skjoth, E. Rattenborg, and C. Enevoldsen. 2000. Integration of research, development, health promotion, and milk quality assurance in the Danish dairy industry. Pages 258-260 in Proc. 9th Symp. Int. Soc. Vet. Epidemiol. Econ., Breckenridge, CO. M. D. Salman, P. Morley, and R. Ruch-Gallie, ed. Int. Soc. Vet. Epidemiol. Econ., Fort Collins, CO.

Best, N., M. Cowles, and S. Vines. 1995. CODA Manual version 0.30. MRC Biostatistics Unit, Cambridge, UK.

Choi, Y., W. O. Johnson, I. A. Gardner, and M. T. Collins. 2006. Bayesian inference for receiver operating characteristic curves in the absence of a gold standard. J. Agric. Biol. Environ. Stat. (accepted) 
Dendukuri, N., and L. Joseph. 2001. Bayesian approaches to modeling the conditional dependence between multiple diagnostic tests. Biometrics 57:158-167.

Faraggi, D. 2003. Adjusting receiver operating characteristic curves and related indices for covariates. Statistician 52:179-192.

Garrett, E. S., E. E. Eaton, and S. Zeger. 2002. Methods for evaluating the performance of diagnostic tests in the absence of a gold standard: A latent class model approach. Stat. Med. 21:1289-1307.

Georgiadis, M. P., W. O. Johnson, I. A. Gardner, and R. Singh. 2003. Correlation-adjusted estimation of sensitivity and specificity of two diagnostic tests. Appl. Stat. 52:63-76.

Greiner, M., D. Pfeiffer, and R. D. Smith. 2000. Principles and practical application of receiver operating characteristic analysis for diagnostic tests. Prev. Vet. Med. 45:23-41.

Hui, S. L., and S. D. Walter. 1980. Estimating the error rates of diagnostic tests. Biometrics 36:167-171.

Jeffreys, H. 1961. Theory of Probability. 3rd ed. Oxford University Press, Oxford, UK.

Joseph, L., T. Gyorkos, and L. Coupal. 1995. Bayesian estimation of disease prevalence and the parameters of diagnostic tests in the absence of a gold standard. Am. J. Epidemiol. 141:263-272.

Kudahl, A., S. S. Nielsen, and J. T. Sørensen. 2004. Relationship between antibodies against Mycobacterium avium subsp. paratuberculosis in milk and shape of lactation curves. Prev. Vet. Med. 62:119-134.

Nielsen, S. S. 2002. Variance components of an enzyme-linked immunosorbent assay for detection of IgG antibodies in milk samples to Mycobacterium avium subspecies paratuberculosis in dairy cattle. J. Vet. Med. B 49:384-387.

Nielsen, S. S., C. Enevoldsen, and Y. T. Gröhn. 2002b. The Mycobacterium avium subsp. paratuberculosis ELISA response by parity and stage of lactation. Prev. Vet. Med. 54:1-10.

Nielsen, S. S., C. Gronbak, J. F. Agger, and H. Houe. 2002a. Maximum-likelihood estimation of sensitivity and specificity of ELISAs and faecal culture for diagnosis of paratuberculosis. Prev. Vet. Med. 53:191-204.

Nielsen, S. S., and N. Toft. 2006. Age-specific characteristics of ELISA and fecal culture for purpose-specific testing for paratuberculosis. J. Dairy Sci. 89:569-579.

Pepe, M. 2003. The Statistical Evaluation of Medical Tests for Classification and Prediction. Oxford University Press, New York, NY.

Rideout, B. A., S. Brown, W. C. Davis, J. M. Gay, R. A. Giannella, M. E. Hines, W. D. Hueston, L. J. Hutchinson, and T. Rouse. 2003. The Diagnosis and Control of Johne's Disease. National Academy Press, Washington, DC.

Robert, C. P., and G. Casella. 2004. Monte Carlo Statistical Methods. 2nd ed. Springer, New York, NY.

Schisterman, E. F., D. Faraggi, and B. Reiser. 2004. Adjusting the generalized ROC curve for covariates. Stat. Med. 23:3319-3331.

Spiegelhalter, D. J., A. Thomas, N. G. Best, and W. R. Gilks. 1995. BUGS: Bayesian Inference Using Gibbs Sampling. Version 0.50. MRC Biostatistics Unit, Cambridge, UK.

van Schaik, G., S. M. Stehman, Y. H. Schukken, C. R. Rossiter, and S. J. Shin. 2003. Pooled fecal culture sampling for Mycobacterium avium subsp. paratuberculosis at different herd sizes and prevalence. J. Vet. Diagn. Invest. 15:233-241.

Wang, C., B. W. Turnbull, Y. T. Gröhn, and S. S. Nielsen. 2006. Nonparametric estimation of ROC curves based on Bayesian models when the true disease state is unknown. Tech. Rep. No. 1445, School of Operations Research and Industrial Engineering, Cornell University, Ithaca, NY.

Youden, W. J. 1950. Index for Rating Diagnostic Tests. Cancer 3:32-35.

Zhou, X.-H., P. Castelluccio, and C. Zhou. 2005. Nonparametric estimation of ROC curves in the absence of a gold standard. Biometrics 61:600-609.

Zweig, M. H., and G. Campbell. 1993. Receiver operating characteristic (ROC) plots: A fundamental evaluation tool in clinical medicine. Clin. Chem. 39:561-577.

\section{APPENDIX: THE MCMC PROCEDURE}

Inferences are based on the posterior distribution given the observed data $\left\{T_{i} ; i=1, \ldots, n\right\},\left\{R_{i} ; i=1, \ldots, n\right\}$ and the prior information. Our objective was to generate realizations from the posterior multivariate distribution, which is done by the Gibbs sampler. The Gibbs sampler successively updates each variable by sampling from its conditional distribution given the current values of other variables. After a large enough number of iterations, under mild conditions, the values of the updated variables thus obtained form a sample from the joint posterior distribution (e.g., Robert and Casella, 2004). Detailed description of one sweep of our MCMC procedure is as follows:

(a) Update disease status $\left\{D_{i} ; i=1, \ldots, n\right\}$. For every $i=1, \ldots, n$,

$$
\begin{aligned}
& \pi\left(D_{i} \mid \bullet\right) \propto\left\{\frac{1}{\sqrt{2 \pi} \sigma_{D}} \exp \left[\frac{-\left(T_{i}-\boldsymbol{\beta}_{D}^{\prime} \cdot \mathbf{Z}_{i}\right)^{2}}{2 \sigma_{D}^{2}}\right]\right.
\end{aligned}
$$

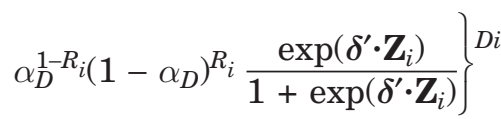

$$
\begin{aligned}
& \times\left\{\frac{1}{\sqrt{2 \pi} \sigma_{H}} \exp \left[\frac{-\left(T_{i}-\boldsymbol{\beta}_{H}^{\prime} \cdot \mathbf{Z}_{i}\right)^{2}}{2 \sigma_{H}^{2}}\right]\right. \\
& \left.\alpha_{H}^{R_{i}}\left(1-\alpha_{H}\right)^{1-R_{i}} \frac{1}{1+\exp \left(\delta^{\prime} \cdot \mathbf{Z}_{i}\right)}\right\}^{1-D_{i}}
\end{aligned}
$$

All these can be sampled from binomial distributions.

(b) Update regression coefficients on means of test scores $\boldsymbol{\beta}_{D}, \boldsymbol{\beta}_{H}$.

$$
\begin{gathered}
\pi\left(\boldsymbol{\beta}_{D} \mid \bullet\right) \propto \prod_{i=1}^{n}\left\{\frac{1}{\sqrt{2 \pi} \sigma_{D}} \exp \left[\frac{-\left(T_{i}-\boldsymbol{\beta}_{D}^{\prime} \cdot \mathbf{Z}_{i}\right)^{2}}{2 \sigma_{D}^{2}}\right] D_{i}\right. \\
\quad \exp \left(\frac{-\boldsymbol{\beta}_{D}^{\prime} \cdot \boldsymbol{\beta}_{D}}{2 \times 1000}\right) \\
\pi\left(\boldsymbol{\beta}_{H} \mid \bullet\right) \propto \prod_{i=1}^{n}\left\{\frac{1}{\sqrt{2 \pi} \sigma_{H}} \exp \left[\frac{-\left(T_{i}-\boldsymbol{\beta}_{H}^{\prime} \cdot \mathbf{Z}_{i}\right)^{2}}{2 \sigma_{H}^{2}}\right]\right\}^{1-D_{i}} \\
\exp \left(\frac{-\boldsymbol{\beta}_{H}^{\prime} \cdot \boldsymbol{\beta}_{H}}{2 \times 1000}\right)
\end{gathered}
$$

Both can be simulated from multivariate normal distributions.

(c) Update variances of test scores $1 / \sigma_{D}^{2}, 1 / \sigma_{H}^{2}$ 


$$
\begin{aligned}
\pi\left(1 / \sigma_{D}^{2} \mid \bullet\right) \propto & \prod_{i=1}^{n}\left\{\frac{1}{\sqrt{2 \pi} \sigma_{D}} \exp \left[\frac{-\left(T_{i}-\boldsymbol{\beta}_{D}^{\prime} \cdot \mathbf{Z}_{i}\right)^{2}}{2 \sigma_{D}^{2}}\right]\right\}_{i} \\
& \left(\frac{1}{\sigma_{D}^{2}}\right)^{0.001-1} \exp \left(-\frac{0.001}{\sigma_{D}^{2}}\right) \\
\pi\left(1 / \sigma_{H}^{2} \mid \bullet\right) \propto & \prod_{i=1}^{n}\left\{\frac{1}{\sqrt{2 \pi} \sigma_{H}} \exp \left[\frac{-\left(T_{i}-\boldsymbol{\beta}_{H}^{\prime} \cdot \mathbf{Z}_{i}\right)^{2}}{2 \sigma_{H}^{2}}\right]\right\}_{i} \\
& \left(\frac{1}{\sigma_{H}^{2}}\right)^{0.001-1} \exp \left(-\frac{0.001}{\sigma_{H}^{2}}\right)
\end{aligned}
$$

Both can be simulated from gamma distributions.

(d) Update misclassification probabilities $\alpha_{D}, \alpha_{H}$

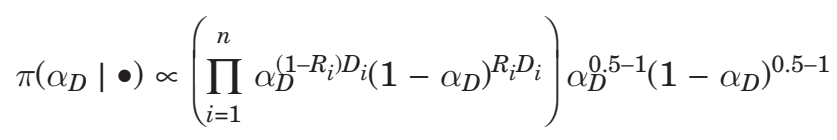

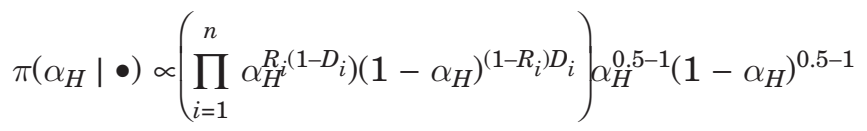

Both can be simulated from beta distributions.

(e) Update regression coefficients $\delta$ on disease prevalence.

$$
\begin{gathered}
\pi(\boldsymbol{\delta} \mid \bullet) \propto \prod_{i=1}^{n}\left[\frac{\exp \left(\boldsymbol{\delta}^{\prime} \cdot \mathbf{Z}_{i}\right)}{1+\exp \left(\delta^{\prime} \cdot \mathbf{Z}_{i}\right)}\right] D_{i}\left[\frac{1}{1+\exp \left(\delta^{\prime} \cdot \mathbf{Z}_{i}\right)}\right] 1-D_{i} \\
\exp \left(\frac{-\delta^{\prime} \cdot \delta}{2 \times 1000}\right)
\end{gathered}
$$

Thus $\delta \mid \bullet$ can be simulated by the Metropolis-Hasting algorithm with a multidimensional random walk. 